PROCEEDINGS OF THE

AMERICAN MATHEMATICAL SOCIETY

Volume 129, Number 5 , Pages 1349-1358

S 0002-9939(00)06032-9

Article electronically published on November 30, 2000

\title{
OSCILLATION INEQUALITIES FOR RECTANGLES
}

\author{
ROGER L. JONES, JOSEPH M. ROSENBLATT, AND MÁTÉ WIERDL
}

(Communicated by Michael Handel)

\begin{abstract}
In this paper we extend previously obtained results on $L^{p}$ norm inequalities $(1<p<\infty)$ for square functions, oscillation and variation operators, with $\mathbb{Z}$ actions, to the case of $\mathbb{Z}^{d}$ actions. The technique involves the use of a result about vector valued maximal functions, due to Fefferman and Stein, to reduce the problem to a situation where we can apply our previous results.
\end{abstract}

\section{INTRODUCTION}

In this paper we consider ergodic square functions and related operators associated with $\mathbb{Z}^{d}$ actions, generalizing certain results obtained in [5] for the case $d=1$. One of the operators we consider, the variation operator, gives us the tools we need to prove inequalities about the number of jumps of size $\lambda$ that occur as the averages converge. It also gives us the tools to prove the exponential estimate of Kalikow and Weiss [8]. Before we discuss the higher dimensional results we give the definitions of the one-dimensional versions. Understanding these will be helpful in the understanding of the higher dimensional analogs.

Let $(X, \Sigma, m, \tau)$ denote a dynamical system with $(X, \Sigma, m)$ a probability space and $\tau$ a measurable invertible measure preserving point transformation from $X$ to itself. Let $A_{n} f(x)=\frac{1}{n} \sum_{k=0}^{n-1} f\left(\tau^{k} x\right)$. Fix an increasing sequence $\left(n_{k}\right)$ and define the square function

$$
S f(x)=\left(\sum_{k=1}^{\infty}\left|A_{n_{k}} f(x)-A_{n_{k+1}} f(x)\right|^{2}\right)^{\frac{1}{2}},
$$

and the oscillation operator

$$
\mathcal{O} f(x)=\left(\sum_{k=1}^{\infty} \sup _{n_{k}<n \leq n_{k+1}}\left|A_{n_{k}} f(x)-A_{n} f(x)\right|^{2}\right)^{\frac{1}{2}} .
$$

Received by the editors July 15, 1999.

2000 Mathematics Subject Classification. Primary 42B25, 28D05; Secondary 40A30.

Key words and phrases. Convergence of ergodic averages, square functions, variation, oscillation, upcrossing inequalities, jump inequalities.

The first author was partially supported by NSF Grant DMS-9531526.

The second author was partially supported by NSF Grant DMS-9705228.

The third author was partially supported by NSF Grant DMS - 9500577 .

(C)2000 American Mathematical Society 
We also consider, for $\varrho>2$, the variation operator

$$
\mathcal{V}_{\varrho} f(x)=\sup _{\left(n_{k}\right)}\left(\sum_{k=1}^{\infty}\left|A_{n_{k}} f(x)-A_{n_{k+1}} f(x)\right|^{\varrho}\right)^{\frac{1}{\varrho}}
$$

where the supremum is taken over all increasing sequences of positive integers. In [5] it is shown that each of these operators is bounded on $L^{p}(X)$ for $1<p<\infty$, and is weak type $(1,1)$. In this paper we show that the higher dimensional analogs, where the averages are taken over rectangles in $\mathbb{Z}^{d}$, are also bounded operators on $L^{p}$ for $1<p<\infty$. We will consider the weak type results, as well as averages over more general regions, in a later paper.

Throughout the paper, $c$ and $C$, sometimes with parameters, will denote positive constants, but their values may be different from one occurrence to the next.

Let $\vec{\tau}=\left(\tau_{1}, \tau_{2}, \ldots, \tau_{d}\right)$ denote a measurable, measure preserving $\mathbb{Z}^{d}$ action. For vectors in $\mathbb{Z}^{d}$ with non-negative coordinate entries, we will use the partial order $\vec{m} \leq \vec{n}$ if each coordinate of $\vec{m}$ is less than or equal to the corresponding coordinate of $\vec{n}$. For $\vec{n}=\left(n_{1}, n_{2}, \ldots, n_{d}\right)$ we will denote by $A_{\vec{n}} f(x)$ the ergodic average

$$
A_{\vec{n}} f(x)=\frac{1}{n_{1} n_{2} \ldots n_{d}} \sum_{j_{1}=0}^{n_{1}-1} \sum_{j_{2}=0}^{n_{2}-1} \cdots \sum_{j_{d}=0}^{n_{d}-1} f\left(\tau_{1}^{j_{1}} \tau_{2}^{j_{2}} \cdots \tau_{d}^{j_{d}} x\right) .
$$

Thus, we are averaging over rectangles in $\mathbb{Z}^{d}$ which have their lower left corner at $(0,0, \ldots, 0)$ and upper right corner at $\vec{n}=\left(n_{1}, n_{2}, \ldots, n_{d}\right)$.

Remark 1.1. While all the results in this paper will be stated and proved in the case of rectangles with "lower left corner" at the origin, the conclusions continue to hold for rectangles that are nested and contain the origin. The modifications required are not difficult, and the necessary $d=1$ case was stated and proved in the required generality in [5].

Remark 1.2. The methods of this paper apply only to averages over a nested sequence of rectangles. They cannot handle the case of nested balls, for example. In a subsequent paper we will consider, via a much different argument, averages over other regions such as balls, and we will be able to prove weak $(1,1)$ bounds as well. Curiously enough, the argument in the subsequent paper requires further restrictions on the sequence of rectangles, and does not give $L^{p}$ bounds for $p>2$.

\section{Square FunCtions}

We first note the following theorem. While more general theorems will be proved later, the basic idea for these more general theorems already appears in this simple case.

Theorem 2.1. Fix a sequence $\left(\vec{n}_{k}\right)$ of vectors in $\mathbb{Z}^{d}$ such that $\vec{n}_{k} \leq \vec{n}_{k+1}$ for each $k$. The square function $S f(x)=\left(\sum_{k=1}^{\infty}\left|A_{\vec{n}_{k}} f(x)-A_{\vec{n}_{k-1}} f(x)\right|^{2}\right)^{\frac{1}{2}}$ is a bounded operator from $L^{p}(X)$ to itself for $1<p<\infty$. In addition, if $f \in L\left(\log ^{+} L\right)^{d}$, then $S f \in L^{1}(X)$.

Remark 2.2. The condition that $\vec{n}_{k} \leq \vec{n}_{k+1}$ implies that we are looking at a family of nested rectangles. It is easy to see that some restriction on the family of rectangles is needed. For example if we consider the case in $\mathbb{Z}^{2}$ where $\vec{n}_{2 k}=(k, 1)$ and $\vec{n}_{2 k+1}=(1, k)$, it is easy to construct examples of $f \in L^{2}$ such that the square 
function diverges a.e. In fact such examples can be constructed even in the onedimensional case, although here, to avoid nested intervals, we need to allow intervals that go both to the left and to the right of zero.

To prove this theorem, as well as later theorems in this paper, we will make use of the following theorem of C. Fefferman and E. M. Stein [3]. (See also [9].)

Theorem 2.3 (Fefferman-Stein). Let $F=\left(f_{1}, f_{2}, \cdots,\right)$ be a sequence of functions on $\mathbb{R}^{d}$. Let $f_{k}^{\star}$ denote the Hardy-Littlewood maximal function of $f_{k}$. If $1<r<\infty$ and $1<p<\infty$, then

$$
\left\|\left(\sum_{k=1}^{\infty}\left|f_{k}^{\star}\right|^{r}\right)^{\frac{1}{r}}\right\|_{p} \leq C(r, p, d)\left\|\left(\sum_{k=1}^{\infty}\left|f_{k}\right|^{r}\right)^{\frac{1}{r}}\right\|_{p} .
$$

Remark 2.4. Actually we need this theorem for function in $\ell^{p}(\mathbb{Z})$, but an examination of the proof shows that the result holds in the discrete setting as well.

For $\sigma$ a measurable measure preserving transformation from $X$ to itself, we define the ergodic maximal function by

$$
M f(x)=\sup _{n>0} \frac{1}{n} \sum_{k=0}^{n-1}\left|f\left(\sigma^{k} x\right)\right| .
$$

By a standard application of the Calderón transfer principle, the discrete version of the Fefferman-Stein Theorem can be stated in the dynamical systems setting. (See 1] or 2] for a discussion of Calderón's transfer principle.) Thus the following result holds.

Theorem 2.5. Let $F=\left(f_{1}, f_{2}, \cdots,\right)$ be a sequence of functions in $L^{p}(X)$. Let $M f_{k}$ denote the ergodic maximal function of $f_{k}$. If $1<r<\infty$ and $1<p<\infty$, then

$$
\left\|\left(\sum_{k=1}^{\infty}\left|M f_{k}\right|^{r}\right)^{\frac{1}{r}}\right\|_{p} \leq C(r, p)\left\|\left(\sum_{k=1}^{\infty}\left|f_{k}\right|^{r}\right)^{\frac{1}{r}}\right\|_{p} .
$$

Before we begin the proof of Theorem 2.1 we introduce some further notation that will also be used in later results. If $\vec{n}=\left(n_{1}, n_{2}, \ldots, n_{d}\right)$, the one-dimensional average in the $k$ th coordinate will be denoted by $A_{\vec{n}}^{k} f(x)$. That is,

$$
A_{\vec{n}}^{k} f(x)=\frac{1}{n_{k}} \sum_{j=0}^{n_{k}-1} f\left(\tau_{k}^{j} x\right) .
$$

The maximal operator in the $k$ th coordinate will be denoted by $M_{k} f(x)$; thus

$$
M_{k} f(x)=\sup _{n} \frac{1}{n} \sum_{j=0}^{n-1}\left|f\left(\tau_{k}^{j} x\right)\right| .
$$

Note that $A_{\vec{n}} f(x)=A_{\vec{n}}^{1} A_{\vec{n}}^{2} \ldots A_{\vec{n}}^{d} f(x)$. Since we have assumed that the $\tau_{j}$ commute, the same is true for the operators $A_{\vec{n}}^{j}, j=1,2, \ldots, d$.

Proof of Theorem [2.1] We will give the complete details in the case of $d=2$. The general case will follow in the same way, with an easy induction argument. The only additional complication is in the notation. 
The idea is to introduce new rectangles in such a way that we still have an increasing family of rectangles, but such that only one side length changes at any stage.

Let $f \in L^{p}(X)$. We have

$$
\begin{aligned}
S f(x)= & \left(\sum_{k=1}^{\infty}\left|A_{\vec{n}_{k}}^{1} A_{\vec{n}_{k}}^{2} f(x)-A_{\vec{n}_{k-1}}^{1} A_{\vec{n}_{k-1}}^{2} f(x)\right|^{2}\right)^{\frac{1}{2}} \\
\leq & \left(\sum_{k=1}^{\infty}\left|A_{\vec{n}_{k}}^{1}\left(A_{\vec{n}_{k}}^{2} f(x)-A_{\vec{n}_{k-1}}^{2} f(x)\right)+A_{\vec{n}_{k-1}}^{2}\left(A_{\vec{n}_{k}}^{1} f(x)-A_{\vec{n}_{k-1}}^{1} f(x)\right)\right|^{2}\right)^{\frac{1}{2}} \\
\leq & \left(\sum_{k=1}^{\infty}\left|A_{\vec{n}_{k}}^{1}\left(A_{\vec{n}_{k}}^{2} f(x)-A_{\vec{n}_{k-1}}^{2} f(x)\right)\right|^{2}\right)^{\frac{1}{2}} \\
& +\left(\sum_{k=1}^{\infty}\left|A_{\vec{n}_{k-1}}^{2}\left(A_{\vec{n}_{k}}^{1} f(x)-A_{\vec{n}_{k-1}}^{1} f(x)\right)\right|^{2}\right)^{\frac{1}{2}} \\
\leq & \left(\sum_{k=1}^{\infty}\left(M_{1}\left|A_{\vec{n}_{k}}^{2} f(x)-A_{\vec{n}_{k-1}}^{2} f(x)\right|\right)^{2}\right)^{\frac{1}{2}} \\
& +\left(\sum_{k=1}^{\infty}\left(M_{2}\left|A_{\vec{n}_{k}}^{1} f(x)-A_{\vec{n}_{k-1}}^{1} f(x)\right|\right)^{2}\right)^{\frac{1}{2}} \\
= & S_{1} f(x)+S_{2} f(x) .
\end{aligned}
$$

Here $M_{1}$ is the one-dimensional maximal operator associated with $\tau_{1}$, and $M_{2}$ is the one-dimensional maximal operator associated with $\tau_{2}$.

We will just handle the operator $S_{1} f(x)$; the operator $S_{2} f(x)$ can be handled similarly.

We have, by an application of the dynamical system version of the FeffermanStein Theorem,

$$
\begin{aligned}
\left\|S_{1} f\right\|_{p}^{p} & =\left\|\left(\sum_{k=1}^{\infty}\left[M_{1}\left|A_{\vec{n}_{k}}^{2} f(x)-A_{\vec{n}_{k-1}}^{2} f(x)\right|\right]^{2}\right)^{\frac{1}{2}}\right\|_{p}^{p} \\
& \leq\left\|\left(\sum_{k=1}^{\infty}\left|A_{\vec{n}_{k}}^{2} f(x)-A_{\vec{n}_{k-1}}^{2} f(x)\right|^{2}\right)^{\frac{1}{2}}\right\|_{p}^{p}
\end{aligned}
$$

Note that the operator

$$
\left(\sum_{k=1}^{\infty}\left|A_{\vec{n}_{k}}^{2} f(x)-A_{\vec{n}_{k-1}}^{2} f(x)\right|^{2}\right)^{\frac{1}{2}}
$$

is just a standard one-dimensional square function, with transformation $\tau_{2}$. We know that the one-dimensional square function is bounded on $L^{p}$ for all $p, 1<p<$ $\infty$. (See [4] or [5] for a proof of this.)

Combining the two observations above, we have the conclusion of the first statement of the Theorem 2.1 in the case of two transformations.

For $d>2$ we use induction. By the results in [4, we know that the result holds for $d=1$. Assume the result holds for $d-1$. Arguing as above, we can again obtain $S_{1} f, S_{2} f, \ldots, S_{d} f$, where for each fixed $t_{j}$, the operator $S_{j}$ is a $d-1$ dimensional square function. By our induction assumption the result follows as in the case $d=2$ above. 
To prove the second claim in the statement of Theorem [2.1, we note that by induction the sublinear operator $S$ satisfies $\|S f\|_{p} \leq \frac{A}{(p-1)^{d}}\|f\|_{p}$ for $1<p \leq 2$. The result is then completed by an application of the following Lemma. The proof of this Lemma (in the case of linear operators) is contained in Zygmund 10, page 119]. It is included here (with small modifications to handle the sub-linear case) for completeness.

Lemma 2.6. Let $T$ be a sublinear operator on $L^{p}(X)$, where $X$ is a probability space. Assume for some integer $d>0$ that $\|T f\|_{p} \leq \frac{A}{(p-1)^{d}}\|f\|_{p}$ for $1<p \leq 2$. Then $\|T f\|_{1} \leq c_{d} \int_{X}|f(x)|\left(\log ^{+}|f(x)|\right)^{d} d x+c_{d}$ where $c_{d}$ is a fixed constant that depends only on the constant $A$ and the integer $d$.

Proof. Write $f_{k}=f \chi_{\left\{2^{k-1}<|f| \leq 2^{k}\right\}}$ for $k \geq 1$, and let $f_{0}=f \chi_{\{0 \leq|f| \leq 1\}}$. Then $\|T f\|_{1} \leq\left\|\sum_{k}\left|T f_{k}\right|\right\|_{1} \leq \sum_{k}\left\|T f_{k}\right\|_{1}$. Let $B_{k}$ denote the support of $f_{k}$. Since we are working on a probability space, we also have for any choice of $p_{k}, 1<p_{k} \leq 2$, that

$$
\left\|T f_{k}\right\|_{1} \leq\left\|T f_{k}\right\|_{p_{k}} \leq \frac{A}{\left(p_{k}-1\right)^{d}}\left\|f_{k}\right\|_{p_{k}}
$$

where the second inequality follows from the hypothesis regarding the $L^{p}$ bounds. Letting $p_{k}=1+\frac{1}{k+1}$, we have

$$
\begin{aligned}
\left\|T f_{k}\right\|_{1} & \leq A(k+1)^{d}\left\|f_{k}\right\|_{\frac{k+2}{k+1}} \\
& \leq A(k+1)^{d} 2^{k} m\left(B_{k}\right)^{\frac{k+1}{k+2}} .
\end{aligned}
$$

Let $K=\left\{k: m\left(B_{k}\right)<\frac{1}{4^{k}}\right\}$. We have

$$
\begin{aligned}
\|T f\|_{1} \leq & \sum_{k}\left\|T f_{k}\right\|_{1} \\
\leq & \sum_{k \notin K} A(k+1)^{d} 2^{k} m\left(B_{k}\right) m\left(B_{k}\right)^{-1 /(k+2)} \\
& +\sum_{k \in K} A(k+1)^{d} 2^{k} m\left(B_{k}\right)^{(k+1) /(k+2)} \\
\leq & \sum_{k \notin K} A(k+1)^{d} 2^{k} m\left(B_{k}\right) 4^{k /(k+2)}+\sum_{k \in K} A(k+1)^{d} 2^{k} 4^{-k\left(\frac{k+1}{k+2}\right)} \\
\leq & 4 A \sum_{k=0}^{\infty}(k+1)^{d} 2^{k} m\left(B_{k}\right)+A \sum_{k=0}^{\infty}(k+1)^{d} 2^{-k / 3} \\
\leq & c_{d} \int_{X}|f(x)|\left(\log ^{+}|f(x)|\right)^{d} d x+c_{d} .
\end{aligned}
$$

\section{OsCiLLATion AND VARIATION OPERATORS}

Fix an increasing sequence of vectors, $\left(\vec{n}_{k}\right)$, and define the oscillation operator $\mathcal{O}$ by

$$
\mathcal{O} f(x)=\left(\sum_{k=1}^{\infty} \sup _{\vec{n}_{k-1} \leq \vec{m} \leq \vec{n}<\vec{n}_{k}}\left|A_{\vec{n}} f(x)-A_{\vec{m}} f(x)\right|^{2}\right)^{\frac{1}{2}}
$$


Define the variational operator $\mathcal{V}_{\varrho}$ by

$$
\mathcal{V}_{\varrho} f(x)=\sup _{\left(\vec{n}_{k}\right)}\left(\sum_{k=1}^{\infty}\left|A_{\vec{n}_{k}} f(x)-A_{\vec{n}_{k-1}} f(x)\right|^{\varrho}\right)^{\frac{1}{\varrho}},
$$

where the supremum is taken over all increasing sequences of vectors in $\mathbb{Z}^{d}$.

Remark 3.1. In our definition of $\mathcal{V}_{\varrho}$ we are considering rectangles with lower left corner at the origin. The argument we will give can be easily modified to cover the case where we select a subsequence from a fixed nested family of rectangles which can be in a more general position than having the lower left corner at the origin.

We note that in [5] it was shown that if $d=1$, then the operator $\mathcal{O}$ satisfies $\|\mathcal{O} f\|_{p} \leq c(p)\|f\|_{p}$ for $1<p<\infty$, and with $\varrho>2$ the operator $\mathcal{V}_{\varrho}$ satisfies $\left\|\mathcal{V}_{\varrho} f\right\|_{p} \leq c(p, \varrho)\|f\|_{p}$ for $1<p<\infty$. We can now prove that for $d \geq 1$ the following theorem holds.

Theorem 3.2. Let $\tau$ be a $\mathbb{Z}^{d}$ action.

(a) Let $\mathcal{O}$ be the oscillation operator based on the increasing sequence $\left(\vec{n}_{k}\right)$. For each $p, 1<p<\infty$, the operator $\mathcal{O}$ satisfies $\|\mathcal{O} f\|_{p} \leq c(p)\|f\|_{p}$.

(b) For each $\varrho>2$, and each $p, 1<p<\infty$, we have $\left\|\mathcal{V}_{\varrho} f\right\|_{p} \leq c(p, d, \varrho)\|f\|_{p}$.

(c) Let $\mathcal{O}$ be the oscillation operator based on the increasing sequence $\left(\vec{n}_{k}\right)$. If $f \in L\left(\log ^{+} L\right)^{d}$, then $\mathcal{O} f \in L^{1}(X)$.

(d) If $\varrho>2$, and $f \in L\left(\log ^{+} L\right)^{d}$, then $\mathcal{V}_{\varrho} f \in L^{1}(X)$.

Proof. Many of the details are the same as in the proof of Theorem 2.1 for the square function. Hence we will only indicate the places where the argument is different.

We will first prove (b) in the case $d=2$. To make sure we consider only measurable functions, we modify $\mathcal{V}_{\varrho}$. Fix a (large) integer $N$ and define the vector $\vec{N}=(N, N, \ldots, N)$. Define

$$
\mathcal{V}_{\varrho, \vec{N}} f(x)=\sup _{\left(\vec{n}_{k} \leq \vec{N}\right)}\left(\sum_{k}\left|A_{\vec{n}_{k}} f(x)-A_{\vec{n}_{k-1}} f(x)\right|^{\varrho}\right)^{\frac{1}{\varrho}},
$$

where the supremum is taken over all increasing sequences of vectors in $\mathbb{Z}^{d}$ satisfying $\vec{n}_{k} \leq \vec{N}$ for each $k$. Since we now have only a finite number of vectors to consider, there is no problem with the measurability of $\mathcal{V}_{\varrho, \vec{N}}$. We will prove (b) for $\mathcal{V}_{\varrho, \vec{N}}$, with the constant independent of $\vec{N}$; then we can let $\vec{N}$ go to infinity. Since $\mathcal{V}_{\varrho, \vec{N}}$ increases to $\mathcal{V}_{\varrho}$, standard arguments imply that $\mathcal{V}_{\varrho}$ is also measurable and satisfies the same norm estimates.

Fix $f \in L^{p}(X)$. For almost every $x \in X$ we can select a (finite) sequence of vectors, $\left(\vec{n}_{k}(x)\right)$, such that

$$
\mathcal{V}_{\varrho, \vec{N}} f(x) \leq 2\left(\sum_{k}\left|A_{\vec{n}_{k}(x)} f(x)-A_{\vec{n}_{k-1}(x)} f(x)\right|^{\varrho}\right)^{\frac{1}{\varrho}} .
$$

We then introduce new rectangles as in the argument for the square function and write $\mathcal{V}_{\varrho, \vec{N}} f(x) \leq 2 V_{\varrho}^{1} f(x)+2 V_{\varrho}^{2} f(x)$ where

$$
V_{\varrho}^{1} f(x)=\left(\sum_{k}\left|A_{\vec{n}_{k}(x)}^{1} A_{\vec{n}_{k}(x)}^{2} f(x)-A_{\vec{n}_{k}(x)}^{1} A_{\vec{n}_{k-1}(x)}^{2} f(x)\right|^{\varrho}\right)^{\frac{1}{\varrho}}
$$


and

$$
V_{\varrho}^{1} f(x)=\left(\sum_{k}\left|A_{\vec{n}_{k}(x)}^{1} A_{\vec{n}_{k-1}(x)}^{2} f(x)-A_{\vec{n}_{k-1}(x)}^{1} A_{\vec{n}_{k-1}(x)}^{2} f(x)\right|^{\varrho}\right)^{\frac{1}{\varrho}} .
$$

Arguing as before, we have

$$
\begin{aligned}
\left\|V_{\varrho}^{1} f\right\|_{p} & =\left\|\left(\sum_{k}\left|A_{\vec{n}_{k}(x)}^{1}\left[A_{\vec{n}_{k}(x)}^{2} f(x)-A_{\vec{n}_{k-1}(x)}^{2} f(x)\right]\right|^{\varrho}\right)^{\frac{1}{\varrho}}\right\|_{p} \\
& \leq\left\|\left(\sum_{k}\left[M_{1}\left|A_{\vec{n}_{k}(x)}^{2} f(x)-A_{\vec{n}_{k-1}(x)}^{2} f(x)\right|\right]^{\varrho}\right)^{\frac{1}{\varrho}}\right\|_{p} \\
& \leq c_{p}\left\|\left(\sum_{k}\left[A_{\vec{n}_{k}(x)}^{2} f(x)-A_{\vec{n}_{k-1}(x)}^{2} f(x)\right]^{\varrho}\right)^{\frac{1}{\varrho}}\right\|_{p} \\
& \leq c_{p}\|f\|_{p} .
\end{aligned}
$$

As before, the next to the last step was an application of Theorem 2.5 (with $r=\varrho$ ) and the last step was an application of the $d=1$ result in [5]. The rest of the argument is no different from that given for the square function.

To prove (a), we argue in a similar way. For almost every $x \in X$ we can select a pair of sequences of vectors $\left(\vec{m}_{k}(x)\right)$ and $\left(\vec{n}_{k}(x)\right)$ such that for each $k$ we have

$$
\vec{n}_{k} \leq \vec{m}_{k}(x)<\vec{n}_{k}(x)<\vec{n}_{k+1},
$$

where $\left(\vec{n}_{k}\right)$ is the sequence (that does not depend on $x$ ) which is used in the definition of $\mathcal{O}$. The argument now proceeds as before.

The proof for (c) and (d) is exactly the same as in the square function case.

\section{UPCROSSING AND JUMP INEQUALITIES}

For any family of operators, $\left(T_{t}\right)$, with a partially ordered index set, and $\lambda>0$, we define $\lambda$-jump operator, $\Lambda\left(\lambda, T_{t}, f, x\right)$, to be the number of $\lambda$ jumps of the family $\left(T_{t} f(x)\right)$, that is, the maximal $N$ for which there are $t_{1}<t_{2}<\cdots<t_{N+1}$ so that for $m=1,2, \ldots, N$ we have

$$
\left|T_{t_{m}} f(x)-T_{t_{m+1}} f(x)\right|>\lambda
$$

In our case we have a family of averaging operators indexed by the vector $\vec{n}$ which determine the "upper right" corner of each averaging rectangle (recall we are considering rectangles with the "lower left" corner at $(0,0, \ldots, 0))$. As before, let $A_{\vec{n}} f(x)$ denote the average over the rectangle associated with $\vec{n}$, and order the vectors by $\vec{m} \leq \vec{n}$ if each coordinate of $\vec{m}$ is less than or equal to the corresponding coordinate of $\vec{n}$. Define $\Lambda\left(\lambda, A_{\vec{n}}, f, x\right)$ to be the maximal $N$ for which there exist $\vec{n}_{1}<\vec{n}_{2} \cdots<\vec{n}_{N+1}$ so that

$$
\left|A_{\vec{n}_{m}} f(x)-A_{\vec{n}_{m+1}} f(x)\right|>\lambda
$$

for $m=1,2, \ldots, N$.

In what follows, we will often abbreviate $\Lambda\left(\lambda, A_{\vec{n}}, f, x\right)$ by $\Lambda(f, x)$.

Theorem 4.1. For each $\varrho, 2<\varrho<\infty$, and each $p, 1<p<\infty$, there is a constant $c(p, \varrho)$ so that for any $f \in L^{p}$, any $\lambda>0$ and positive integer $r$, we have

$$
m\left\{x: \Lambda\left(\lambda, A_{\vec{n}}, f, x\right)>r\right\} \leq \frac{C(p, \varrho)}{\lambda^{p} r^{p / \varrho}}\|f\|_{p}^{p} .
$$


Proof. Note that $\lambda\left(\Lambda\left(\lambda, A_{\vec{n}}, f, x\right)\right)^{\frac{1}{\varrho}} \leq V_{\varrho} f(x)$. Consequently,

$$
\begin{aligned}
m\left\{x: \Lambda\left(\lambda, A_{\vec{n}}, f, x\right)>r\right\} & =m\left\{x: \lambda\left(\Lambda\left(\lambda, A_{\vec{n}}, f, x\right)\right)^{\frac{1}{\varrho}}>\lambda r^{\frac{1}{\varrho}}\right\} \\
& \leq m\left\{x: V_{\varrho} f(x)>\lambda r^{\frac{1}{\varrho}}\right\} \\
& \leq \frac{c(\varrho, p)}{\left(\lambda r^{\frac{1}{\varrho}}\right)^{p}}\|f\|_{p}^{p},
\end{aligned}
$$

where the last step used Chebyshev's inequality and the fact that $\mathcal{V}_{\varrho}$ is a bounded operator from $L^{p}$ to $L^{p}$ for $1<p<\infty$.

Remark 4.2. Note that in the above theorem we are not restricted to a single family of rectangles. Indeed, the only restriction on the rectangles (besides having their "lower left" corner at the origin) is that for each $x$ and $f$, the nested sequences of rectangles is chosen in a measurable way.

Fix an increasing sequence of vectors, $\left(\vec{n}_{k}\right)$, and recall the definition of upcrossings. Fix $\beta>\alpha$. Let $N(f, x)=N(\alpha, \beta, f, x)$ be the number of upcrossings of the sequence $\left(A_{\vec{n}} f(x)\right)$, that is, the maximal $N$ for which there are $s(1)<t(1)<$ $s(2)<t(2)<\cdots<s(N)<t(N)$ so that $A_{\vec{n}_{s(i)}} f(x)<\alpha$ and $A_{\vec{n}_{t(i)}} f(x)>\beta$ for $i=1,2, \ldots, N$.

Of course, Theorem 4.1 has the following corollary for upcrossings.

Theorem 4.3. For each $\varrho, 2<\varrho<\infty$, and each $p, 1<p<\infty$, there is a constant $C(p, \varrho)$ so that for any $f \in L^{p}$, any $\beta>\alpha$ and positive integer $r$, we have

$$
m\{x: N(\alpha, \beta, f, x)>r\} \leq \frac{C(\varrho, p)}{\left((\beta-\alpha) r^{\frac{1}{\varrho}}\right)^{p}}\|f\|_{p}^{p} .
$$

In the case of the family of rectangles given by $R_{n}=(-n, n) \times(-n, n)$, Kalikow and Weiss $[8]$ prove

$$
m\left\{x: \Lambda\left(\lambda, A_{k}, f, x\right)>n\right\} \leq \frac{C}{\lambda} \frac{(\log n)^{3}}{n^{1 / 3}}\|f\|_{1} .
$$

Our result is in some ways an improvement of their result, and in some ways is not as good. Because of the techniques we are using, we cannot expect to get the result for $f \in L^{1}(X)$. However, we see that the term $\frac{(\log n)^{3}}{n^{1 / 3}}$ can be improved to as close to $\frac{c(\varrho, p)}{\sqrt{n}}$ as desired, by simply taking taking $\varrho$ close to 2 and $p$ close to 1. Of course there is a price to pay. Each time we move closer to $\sqrt{n}$ we need to increase the constant $c(\varrho, p)$. (Improvement of the term involving $n$ beyond $\frac{c}{\sqrt{n}}$ is impossible, even in the case $d=1$. See [7].)

In terms of the kind of rectangles considered, their result is more restricted than ours. While their proof is given in the case of squares, as they state, their result will continue to hold if they use families of rectangles with the ratio of the side lengths bounded. However, it seems that their proof does not apply to an arbitrary nested sequences of rectangles.

In our case we do not need to place any restriction on the ratio of the side lengths. In [6], with additional restrictions on the family of rectangles, and quite different techniques, we can obtain the "correct estimate", $\frac{c}{\sqrt{n}}$. 
Next, we turn to the exponential estimate for upcrossings. In [5] we proved that if there is a nontrivial estimate for the upcrossings for bounded functions, then we have an exponential estimate for upcrossings for bounded functions. Although in that paper we applied the result only to operators associated with $\mathbb{Z}$ actions, we stated and proved it for $\mathbb{Z}^{d}$ actions.

Theorem 4.4 ([5]). Let $R_{1}, R_{2}, \ldots$ be a sequence of rectangles in $\mathbb{Z}^{d}$ so that each rectangle contains the origin and the sequence of side lengths in each coordinate direction is non-decreasing (for example, a nested sequence of rectangles). Suppose that for every pair $(\alpha, \beta)$ of numbers with $\alpha<\beta$, there is a function $\epsilon(q)=\epsilon(\alpha, \beta, q)$ so that:

(1) $\lim _{q \rightarrow \infty} \epsilon(q)=0$.

(2) For every function $f: \mathbb{Z}^{d} \rightarrow \mathbb{R}$ of finite support and $\|f\|_{\ell^{\infty}\left(Z^{d}\right)} \leq 1$, we have

$$
|\{x: N(f, x) \geq q\}| \leq \epsilon(q) \cdot\|f\|_{\ell^{1}\left(Z^{d}\right)} .
$$

Then for every pair $(\alpha, \beta)$ of numbers with $\alpha<\beta$, there are positive constants $C=$ $C(\alpha, \beta)$ and $c=c(\alpha, \beta)$, with $c<1$, so that for any dynamical system $(X, \Sigma, m)$ and function $f: X \rightarrow \mathbb{R}$, with $\|f\|_{L^{\infty}(X)} \leq 1$, we have

$$
m(x: N(f, x) \geq n) \leq C \cdot c^{n} \cdot\|f\|_{L^{1}(X)} .
$$

Consequently, we have the following result, which relates to the result of Kalikow and Weiss [8].

Theorem 4.5. If $\left(A_{n}\right)$ is a family of averages associated with a nested sequence of rectangles in $\mathbb{Z}^{d}$, then for all $f \in L^{\infty}$ with $\|f\|_{\infty} \leq 1$, we have the estimate

$$
m\{x: N(f, x) \geq n\} \leq C \cdot c^{n} \cdot\|f\|_{L^{1}(X)} .
$$

Proof. With standard transferance arguments, Theorem 4.3 implies the necessary hypothesis for $f: \mathbb{Z}^{d} \rightarrow \mathbb{R}$ to enable us to apply Theorem 4.4. We apply Theorem 4.4 and we are done.

The following corollary of Theorem 4.5 is almost immediate from the pigeon hole principle.

Corollary 4.6. Let $\lambda>0$. With the assumptions of Theorem 4.4, there are positive constants $C=C(\lambda)$ and $c=c(\lambda)$, with $c<1$, so that for any dynamical system $(X, \Sigma, m)$ and function $f: X \rightarrow \mathbb{R}$, with $\|f\|_{L^{\infty}(X)} \leq 1$, we have

$$
m\{x \mid \Lambda(f, x) \geq n\} \leq C \cdot c^{n} \cdot\|f\|_{L^{1}(X)} .
$$

Proof. Fix $0<\lambda<2$, and let $\lambda_{0}=\lambda / 2$. Let $r=\left[1 / \lambda_{0}\right]+1$, and for $i=-r,-r+$ $1, \ldots, r-1$ let $\alpha_{i}=i \lambda_{0}$ and $\beta_{i}=(i+1) \lambda_{0}$. For each of these $2 r$ intervals we have the exponential estimate of Theorem 4.5 Next, note that by replacing $f$ by $-f$, each upcrossing becomes a downcrossing for the reflected interval. Hence we have an estimate for the number of downcrossings. For each pair $\left(\vec{n}_{m}, \vec{n}_{m+1}\right)$ that satisfies $\left|A_{\vec{n}_{m}} f(x)-A_{\vec{n}_{m+1}} f(x)\right|>\lambda$, we either have an upcrossing or downcrossing of one of our $2 r$ intervals $\left(\alpha_{i}, \beta_{i}\right)$. Since we have exponential control of the upcrossings and downcrossings of each of these finite number of intervals, we have exponential control of the number of $\lambda$-jumps. 


\section{REFERENCES}

1. Bellow, A., Transfer principles in ergodic theory, in Harmonic Analysis and Partial Differential Equations, Chicago Lectures in Mathematics, M. Christ, C. Kenig and C. Sadosky ed., University of Chicago Press, Chicago 1999, pages 27-39.

2. Caledrón, A. P., Ergodic theory and translation invariant operators, Proc. Nat. Acad. of Sci., USA 59 (1968) 349-353. MR 37:2939

3. Fefferman, C. and Stein, E. M., Some maximal inequalities Amer. J. Math. 93 107-115. MR 44:2026

4. Jones, R. L., Ostrovskii, I. and Rosenblatt, J. Square functions in ergodic theory, Ergod. Th. \& Dynam. Sys., 16 (1996) 267-305. MR 97f:28044

5. Jones R. L., Kaufman, R., Rosenblatt, J. and Wierdl, M. Oscillation in ergodic theory, Ergod. Th. \& Dynam. Sys., 18 (1998) 889-935. MR 2000b:28019

6. Jones, R.L., Rosenblatt, J. and Wierdl, M., Oscillation inequalities, the higher dimensional case, preprint.

7. Jones, R.L., Rosenblatt, J. and Wierdl, M., Counting in ergodic theory, Canad. J. Math., 51 (1999) 996-1019. MR 2000i:28021]

8. Kalikow, S. and Weiss, B., Fluctuations of ergodic averages, Il. J. Math., 43 (1999) 480-488. CMP 99:17

9. Stein, E.M., Harmonic Analysis, Princeton University Press, Princeton, NJ, 1993. MR 95c:42002

10. Zygmund, A., Trigonometric Series, (Vol II), Cambridge University Press, Cambridge, 1968. MR 38:4882

Department of Mathematics, DePaul University, 2320 N. Kenmore, Chicago, Illinois 60614

E-mail address: rjones@condor.depaul.edu

Department of Mathematics, University of Illinois at Urbana, Urbana, Illinois 61801

E-mail address: jrsnbltt@symcom.math.uiuc.edu

Department of Mathematical Sciences, University of Memphis, Memphis, Tennessee 38152

E-mail address: wierdlm@mathsci.msci.memphis.edu 